\section{Cancers ORL et génomique}

\section{Gérard Milano}

> Les cancers ORL en France - au quatrième rang après les cancers du sein, de la prostate et du côlon - représentent un important problème de santé publique. La survie à cinq ans plafonne en dépit de progrès thérapeutiques indéniables et d'une prise en charge thérapeutique en rapide évolution, notamment grâce aux traitements ciblés associés aux cytotoxiques conventionnels. Les études en cours permettent encore d'espérer de nouveaux marqueurs biologiques prédictifs et pronostiques, mais la complexité de ces paramètres, ainsi que les besoins d'équipements lourds et onéreux pour les explorer, nécessitent une unification des efforts et des moyens. C'est l'objectif majeur du programme coopératif PACOR. <

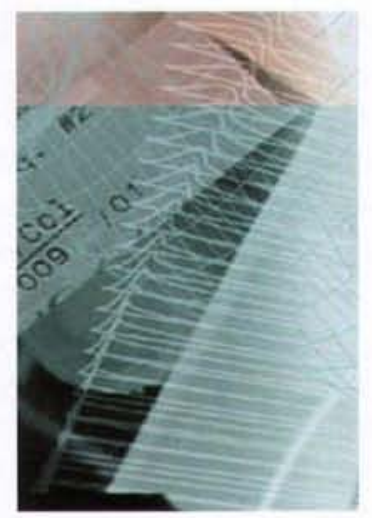

Laboratoire d'Oncopharmacologie EA 3836 et CRLCC de Nice, Centre Antoine Lacassagne, 33, avenue de Valombrose, 06100 Nice, France.
Les cancers ORL sont essentiellement les cancers des voies aérodigestives supérieures (VADS) développés aux dépens de la cavité buccale, du pharynx, du larynx et des cavités nasosinusiennes.

Ils représentent environ $20 \%$ de la totalité des cancers humains.

Les cancers de la cavité buccale, de l'oropharynx, de I'hypopharynx et du larynx frappent essentiellement I'homme ( $95 \%$ des cas), leur maximum de fréquence se situe entre 45 et 70 ans. Ils sont dus essentiellement à l'association de deux substances irritantes: tabac et alcool.

L'histologie de ces cancers met en évidence le plus souvent des carcinomes (épithéliomas) malpighiens plus ou moins différenciés. Ils s'accompagnent souvent d'adénopathies. II faut toujours rechercher un cancer associé ( $20 \%$ des cancers ORL - cavité buccale, oropharynx, hypopharynx, larynx sus-glottique - sont associés à un cancer bronchique). C'est dire l'intérêt du bilan pré-thérapeutique (panendoscopie) et du suivi post-thérapeutique.

Les métastases pulmonaires, hépatiques, osseuses, cérébrales étaient classiquement peu fréquentes : elles le deviennent actuellement puisque l'évolution après traitement est plus longue. Néanmoins, la survie excède rarement cinq ans.
Les cancers ORL en France se situent par leur importance au quatrième plan après les cancers du sein, de la prostate et du côlon. Avec 6000 décès annuels en moyenne, ils représentent un important problème de santé publique. La survie à cinq ans plafonne en dépit de progrès thérapeutiques qui restent indéniables.

\section{Recherche des marqueurs}

Les avancées récentes dans la prise en charge des cancers ORL reposent en grande partie sur les résultats de la recherche clinico-biologique et sur le transfert thérapeutique.

La recherche clinico-biologique a permis, grâce à l'identification de marqueurs biologiques de mieux définir les patients à risque, d'évaluer la sensibilité thérapeutique aussi bien en radiothérapie qu'en chimiothérapie.

C'est ainsi que, dès 1996, la forte valeur pronostique du récepteur du facteur de croissance épidermique (EGFR) a été démontrée $[1,2]$.

Une des difficultés actuelles est de pouvoir combiner ces stratégies et de trouver les meilleures séquences, en particulier pour l'association chimiothérapie-radiothérapie. Le traitement de la maladie avancée, qu'elle soit locale ou métastatique, pose également encore problème. Quant aux thérapeutiques ciblées, les premiers résultats sont encourageants, mais des progrès restent encore à accomplir. 


\section{Le récepteur du facteur de croissance épidermique (દGFR)}

Des mutations dans le gène suppresseur de tumeurs $p 53$ ont été impliquées par certains auteurs dans la pathogénie des cancers de la tête et du cou. Toutefois, l'ensemble des études donne des résultats discordants, aussi bien en ce qui concerne l'évolution clinique que dans la réponse à la radiothérapie et à la chimiothérapie.

$\varepsilon$ n revanche, les travaux de Shin et al. ont démontré l'implication de I'EGFR dans les étapes de la tumorigenèse des carcinomes épidermoïdes des voies aérodigestives supérieures (VADS) [3]. Les auteurs ont analysé par immunohistochimie 36 tumeurs épithéliales ORL avec étude de tissu dans des sites différents allant de l'épithélium normal à la tumeur avérée en passant par des zones adjacentes pré-malignes. En première observation, les auteurs ont noté une intensité de marquage doublée dans l'épithélium «normal » adjacent aux tumeurs par rapport à l'épithélium témoin provenant de sujets non cancéreux. Par ailleurs, ils ont mentionné une augmentation constante de l'expression de l'EGFR au cours de la progression hyperplasie-dysplasie-carcinome $(p=0,01)$. Il est important de souligner à ce stade que l'EGFR revêt un intérêt comme cible thérapeutique, non seulement au niveau des tumeurs en place mais, comme cela sera abordé plus loin, également comme cible potentielle pour la chimioprévention.

Ishitoya et al. [4] ont examiné une série de 21 tumeurs et ont mis en évidence que la surexpression de l'EGFR touchait $53 \%$ des lésions tumorales, alors que l'amplification génique n'était observée que dans $19 \%$ de ces mêmes tumeurs. II y a donc, pour l'EGFR, dissociation entre surexpression et amplification génique, ce dernier phénomène étant de fréquence nettement inférieure par rapport à la surexpression.

L'équipe ORL du Centre Antoine Lacassagne (CAL, Nice) s'est particulièrement intéressée à l'EGFR comme marqueur pronostique pour le suivi des carcinomes ORL. En 1993, l'étude publiée par Dassonville et al. [1] démontre la valeur pronostique de l'EGFR, qui sera confirmée ultérieurement par une investigation comparable menée par Maurizi et al. en 1996. [2]. L'étude du CAL concernait 109 sujets. L'expression de l'EGFR était déterminée par la technique «radio-ligand» afin d'effectuer des mesures spécifiques et quantitatives de l'EGFR dans les cellules tumorales. Cette expression était extrêmement variable entre les tumeurs explorées avec une médiane à $71 \mathrm{fmol} / \mathrm{mg}$ protéine et des extrêmes allant de 2 à $2302 \mathrm{fmol} / \mathrm{mg}$ protéine. L'évolution de la maladie était étroitement liée à une augmentation des concentrations intra-tumorales de l'EGFR. Pour les tumeurs traitées par chimiothérapie d'induction à base de 5 fluoro-uracile et de cisplatine, le taux d'EGFR intratumoral pré-thérapeutique n'avait pas de valeur prédictive sur l'efficacité de ce traitement de première intention. En revanche, une surexpression de l'EGFR était associée à un raccourcissement significatif de la survie sans événement $(p=0,015)$ et de la survie globale $(p=0,028)$.

Dans une analyse multivariée incluant de nombreux facteurs biologico-cliniques, les deux seuls paramètres pronostiques valables et indépendants furent l'EGFR intratumoral et le stade tumoral [5].

Dans son analyse de la valeur pronostique de l'EGFR, l'équipe de Grandis a intégré la prise en compte de l'expression du ligand spéci- fique, le TGF $\alpha$ [6]. Cette étude concernait un groupe de 91 patients traités par chirurgie et dont la tumeur primitive avait été analysée pour son niveau d'expression en EGFR et TGF $\alpha$ (mesuré par immunohistochimie). Elle a permis de conclure que, non seulement l'EGFR $(p=0,0001)$, mais également le TGF $\alpha(p=0,0001)$, étaient des marqueurs pronostiques de survie sans événement et de survie spécifique. En analyse multivariée, ces deux marqueurs avaient une valeur pronostique indépendante et permettaient une meilleure prédiction de survie que chacun des marqueurs pris isolément.

En 1999, nous avons également comparé la valeur pronostique de la protéine $p 53$ et de l'EGFR dans les cancers ORL [7]. Cette étude a porté sur un total de 82 patients. L' EGFR était déterminé par méthode spécifique avec radio-ligand et l'expression de $p 53$ tumorale était mesurée par technique immunoluminométrique. Par analyse univariée, p53 et EGFR apparaissaient comme des marqueurs pronostiques significatifs mais, en analyse multivariée, seuls l'EGFR et le stade tumoral demeuraient des marqueurs pronostiques indépendants.

L'EGFR représente donc un paramètre doté d'une valeur pronostique indépendante incontestable, non seulement en ce qui concerne le traitement, mais également la chimio-prévention.

\section{Les marqueurs de néoangiogenèse}

L'origine moléculaire de la néoangiogenèse tumorale est complexe et repose en grande partie sur l'émission de signaux tumoraux. Parmi ces signaux, le mieux étudié est celui de la sécrétion du VEGF, sous le contrôle principal de l'EGFR. II est également influencé par le facteur de transcription HIF- $1 \alpha$, reflet de l'état hypoxique tumoral [8]. Un lien entre l'expression tumorale de VEGF (mesuré par immunohistochimie) et le pronostic a été mis en évidence récemment par Uehara et al. [9] sur une série relativement limitée de 63 patients. Beasley et al. [10] ont examiné la relation entre l'expression de HIF-l $\alpha$ et la survie. Ils ont conclu à une valeur pronostique forte (79 cas) de l'expression d'HIF-l $\alpha$, aussi bien pour la survie sans événement que la survie globale. Toutefois, des travaux sont encore nécessaires pour établir la valeur pronostique de l'ensemble des marqueurs d'angiogenèse dans les cancers ORL.

\section{Analyse génomique étendue, à la recherche de nouveaux marqueurs}

Les techniques actuelles d'exploration globale du génome devraient permettre de trouver de nouveaux marqueurs liés au pronostic et à la réponse thérapeutique des cancers $\mathrm{ORL}$. 
Trois explorations récentes méritent d'être signalées:

- le travail de Ginos et al. [11] a conduit à identifier un groupe de gènes codant pour des indicateurs de prolifération cellulaire, des protéines de la matrice extracellulaire, des cytokines, des chimiokines et des protéines impliquées dans la réponse immune qui sont liées à l'évolution des tumeurs;

- l'étude de Chung et al. [12] a abouti à la caractérisation d'un profil multigène de la tumeur primitive qui permet de prévoir les métastases ganglionnaires avec $80 \%$ de sensibilité ;

- enfin, par la technique CGH-array (hybridation génomique comparative), l'analyse de Wreesman et al. [13] a mis en évidence des modifications (perte/acquisition) chromosomiques tumorales. Sur 82 cancers ORL, 5 anomalies chromosomiques à valeur pronostique ont été trouvées, avec deux amplifications 11 q13 et $12 q 24$ et des pertes en $5 q 11,6 q 14$ et $21 q 11$.

L'utilisation des outils de l'analyse génomique étendue permet non seulement d'entrevoir une nouvelle catégorisation des tumeurs ORL selon leur signature génétique, mais aussi d'identifier de nouvelles cibles thérapeutiques potentiellement exploitables.

\section{Le programme coopératif PACOR}

PACOR (PACA-ORL) est un réseau multidisciplinaire centré sur la cancérologie ORL, la chirurgie, la radiothérapie, l'oncologie médicale ainsi que la biopathologie, et qui s'appuie sur deux pôles ORL, Marseille et Nice (Figure 1).

PACOR recouvre $70 \%$ des cancers $O R L$ dans la région PACA soit à peu près $10 \%$ des cancers $O R L$ en France.
II comprend des plateaux techniques de haut niveau: protéomique sur Marseille-Nord et DNA-array à I'IPMC (Institut de Pharmacologie Moléculaire et Cellulaire, Valbonne).

De création récente (novembre 2004), il a pour objectif d'améliorer la connaissance biologique aussi bien du point de vue prédictif et/ou pronostique. II a également l'ambition de rassembler des essais thérapeutiques fédérateurs, tels que "Carissa», essai consistant à associer un inhibiteur de l'EGFR - Iressa ${ }^{\circ}$ - avec le traitement radiothérapique.

PACOR a aussi pour but mettre en œuvre des initiatives nationales provenant du cancéropôle PACA, de la Ligue contre le cancer ou de I'ARC. II comporte des partenariats privilégiés avec l'industrie comme dans le cas de l'essai Carissa.

PACOR se situe à l'interface avec des groupes de travail déjà en place : le GORTEC (groupe oncologie-radiothérapie-tête et cou) et le GETTEC (groupement d'étude des tumeurs de la tête et du cou).

Le premier projet collaboratif initié par PACOR a pour objectif d'aborder le cas particulier posé par les cancers ORL sans facteurs de risque (absence d'exposition à l'alcool et au tabac).

En effet, un certain nombre cancers ORL ( $6 \%$ en moyenne) se développent sans ces facteurs de risques alcoolotabagiques et ont une évolution souvent très défavorable. II est donc important de mettre en évidence les éléments de déréglement génétique ou biologique à l'origine de ces cancers. Ce projet comporte deux volets:

- une démarche cognitive visant à rechercher des paramètres liés à l'angiogenèse, et à l'invasion incluant l'EGFR, ainsi que l'impact de la composante virologique (HPV) (demande de PHRC 2005);

- un programme de génomique descriptive construit dans le cadre du Cancéropôle PACA qui associe deux platesformes, l'une de protéomique qui est en place dans l'unité de Pierre-Marie Martin (Médecine Nord, Marseille) et l'autre de génomique avec l'analyse du transcriptome coordonnée par Pascal Barbry à I'IPMC (Sophia-Antipolis) (projet de Réseau Structurant, appel d'offres 2005 de l'Institut National du (ancer).

\section{Conclusion}

La prise en charge thérapeutique des cancers ORL évolue rapidement, notam-

Figure 1. Organigramme du réseau PACOR (PACA-ORL). 
ment grâce à l'introduction des thérapeutiques ciblées associées aux cytotoxiques conventionnels. Mais les études en cours permettent encore d'espérer de nouveaux marqueurs biologiques prédictifs et pronostiques. Toutefois, la complexité de ces paramètres, ainsi que les besoins d'équipements lourds et onéreux pour les explorer, nécessitent une unification des efforts et des moyens. C'est l'objectif majeur du rapprochement PACOR qui devra faire ses preuves dans cette perspective. $\diamond$

\section{SUMMARY}

\section{ENT cancers and genomics}

ENT cancers in France - with the fourth rank after the breast, colon and prostate localizations - represent an important problem of public health. Survival at five years reaches a maximum in spite of undeniable therapeutic progress, in particular with the targeted treatments associated to conventional cytotoxic drugs. The studies in progress still make it possible to hope for new predictive and prognostic biological markers, but the complexity of these parameters, as well as the needs for heavy and expensive equipment to explore them, require a unification of the efforts and means. It is the major objective of the cooperative program PACOR. $\triangle$

\section{RÉFÉRENCES}

1. Dassonville 0, Formento $\mathrm{JL}$, Francoual $M$, et al. Expression of epidermal growth factor receptor and survival in upper aerodigestive tract cancer. J Clin Oncol 1993; 11:1873- 8 .

2. Maurizi M, Almadori G, Ferrandina G, et al. Pronostic significance of epidermal growth factor receptor in laryngeal squamous cell carcinoma. Br J Cancer 1996; 74:1253-7.

3. Shin DM, Ro Jy, Hong WK, et al. Dysregulation of epidermal growth factor receptor expression in premalignant lesions during head and neck tumorigenesis. Cancer Res $1994 ; 54: 3153$-9.

4. Ishitoya J, Toriyama M, Oguchi N, et al. Gene amplification and overexpression of EGF receptor in squamous cell carcinomas of the head and neck. Br J Cancer $1989 ; 59: 559-62$.

5. Santini J, Formento JL, Francoual M, et al Characterization, quantification, and potential clinical value of the epidermal growth factor receptor in head and neck squamous cell carcinomas. Head Neck $1991 ; 13: 132-9$
6. Grandis JR, Methem MF, Gooding W, et al Levels of TGF- $\alpha$ and EGFR protein in head and neck squamous cell carcinoma and patient survival. I Natl Cancer Inst 1998; $90: 824-32$.

7. Etienne MC, Pivot W, Formento JL, et al. A multivariate approach including tumoral epidermal growth factor receptor, $p 53$, thymidylate synthase and dihydropyrimidine dehydrogenase to predict treatment outcome in head and neck cancer patients receiving 5 -fluorouracil. Br) Cancer $1999 ; 79: 1864-9$.

8. Ferrara N. The role of VEGF in the regulation of physiological and pathological angiogenesis. EXS 2005; $94 ; 209-31$.

9. Uehara M, Sano K, Ikeda $H$, et al. Expression of vascular endothelial growth factor and prognostic oral squamous cell carcinoma. Oral Oncol $2004 ; 40: 321-5$.

10. Beasley NJ, Leek R, Alam M, et al. Hypoxia-inducible factors HIF-lalpho and HIF-2alpha in head and neck cancer: relationship to tumor biology and treatment outcome in surgically resected patients. Cancer Res 2002; $62: 2493-7$

11. Ginos MA, Page GP, Michalowicz BS, et al. Identification of a gene expression signature associated with recurrent disease in squamous cel carcinoma of the head and neck. Cancer Res $2004 ; 64: 55-63$.

12. Chung CH, Parker JS, Karaca G, et al. Molecular classification of head and neck squamous cell carcinomas using patterns of gene expression. Cancer Cell $2004 ; 5 ; 489-500$

13. Wreesmann VB, Shi W, Thaler HT. Identification of novel prognosticators of outcome in squamous cell carcinoma of the head and neck. J Clin Oncol $2004 ; 22: 3965-72$.
TIRÉS À PART

G. Milano

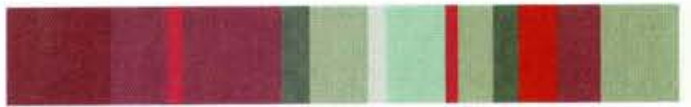

